\title{
Water-soluble pyrene tags enable the detection of carbohydrates by label-assisted laser desorption/ionisation mass spectrometry
}

Jacob R. Hauser, ${ }^{1,2}$ Edmund T. Bergström, ${ }^{3,4}$ Alexander N. Kulak, ${ }^{1}$ Stuart L. Warriner, ${ }^{1,2}$ Jane ThomasOates, ${ }^{3,4}$ and Robin S. Bon *,2,5

${ }^{1}$ School of Chemistry, University of Leeds, Woodhouse Lane, Leeds LS2 9JT, UK.

${ }^{2}$ Astbury Centre for Structural Molecular Biology, University of Leeds, Woodhouse Lane, Leeds LS2 9JT, UK.

${ }^{3}$ Department of Chemistry, University of York, Heslington, York YO10 5DD, UK.

${ }^{4}$ Centre of Excellence in Mass Spectrometry, University of York, Heslington, York YO10 5DD, UK.

${ }^{5}$ Discovery and Translational Science Department, Leeds Institute of Cardiovascular and Metabolic Medicine, LIGHT laboratories, University of Leeds, Leeds LS2 9JT, UK.

Correspondence should be addressed to R.S.B. (r.bon@leeds.ac.uk)

\begin{abstract}
Matrix-assisted laser desorption/ionisation mass spectrometry (MALDI-MS) is widely used for the analysis of biomolecules. Label-assisted laser desorption/ionisation mass spectrometry (LALDI-MS) is a matrix-free variant of MALDI-MS, in which only analytes covalently attached to a laser desorption/ionisation (LDI) enhancer are detected. LALDI-MS has shown promise in overcoming limitations of MALDI-MS in terms of sample preparation and MS analysis. In this work, we have developed water-soluble pyrene-based LDI reagents (LALDI tags) that can be used for (in situ) labelling and LALDI-MS analysis of reducing carbohydrates from complex (biological) samples without the need for additional chemical derivatisation or purification. We have systematically explored the suitability of four pyrene-based LDI enhancers and three aldehyde-reactive handles, optimised sample preparation, and exemplified the use of a LALDI tag by the detection of lactose in cow's milk. These results demonstrate that LALDI-MS is a promising technique for the analysis of reducing carbohydrates in biological samples, and pave the way for the development of LALDI-MS for glycomics and diagnostics.
\end{abstract}

\section{Introduction}

Matrix-assisted laser desorption/ionisation (MALDI) is a soft ionisation technique for mass spectrometry (MS), in which analytes are co-crystallised with a suitable matrix that facilitates absorption of laser light as well as transfer of energy and charge to analytes. ${ }^{1,2}$ MALDI-MS is a powerful analytical tool for studying high molecular weight biomolecules, such as oligonucleotides, lipids, and glycoconjugates. ${ }^{3}$ Because of their low limits of detection and high-throughput capabilities, MALDIMS instruments are increasingly being exploited as platforms for glycomics, proteomics, and point-ofcare diagnostics. ${ }^{4-7}$ Despite the advantages and wide-spread use of MALDI-MS, the presence of a matrix also brings with it several disadvantages: analytes need to be able to mix and co-crystallise with the matrix, co-crystallised samples can contain significant inhomogeneities ("hot spots") leading to challenges with reproducibility and quantification, and matrix peak charge-sharing clusters can obscure low molecular weight (below $\sim 800-1000$ Da) analytes. ${ }^{8}$ Moreover, because of the indiscriminate desorption/ionisation of species present in the matrix, contaminants such as salts, detergents, or more-readily ionised species can result in complicated spectra and analyte signal suppression, meaning purification of analytes may be needed before MALDI-MS analysis. ${ }^{9}$ Analysis of biological species can be especially difficult because reagents commonly used in biological studies, 
such as buffers, salts and detergents, can affect both the co-crystallisation and ionisation. ${ }^{10} \mathrm{High}$ salt concentrations can completely obscure or suppress analyte ionisation, necessitating the use of volatile buffers or sample clean up prior to MALDI-MS analysis. These limitations have prompted the development of various matrix-free LDI-MS approaches. ${ }^{11} \mathrm{~A}$ particularly promising approach is labelassisted laser desorption/ionisation mass spectrometry (LALDI-MS), in which chemical tags (LDI enhancers) cause the selective desorption/ionisation from complex mixtures of the analytes to which they are attached, without the need for purification or an external matrix. ${ }^{11}$

Pyrenes have proven particularly suitable as LDI-enhancing tags. Amano et al. analysed oligosaccharides and glycopeptides derivatised with pyrenebutanoic hydrazide or 1-pyrenyldiazomethane by MALDI-MS. ${ }^{12-15}$ In these experiment, analytes were first purified and then labelled in organic solvent on the MALDI target plate, before co-crystallisation with a matrix. In contrast, Kozmin et al. demonstrated the direct detection of pyrene-labelled small molecules, as their stable (radical) cations, by matrix-free LDI in a MALDI-MS instrument equipped with a standard 355 nm laser. ${ }^{16}$ This enabled high-throughput reaction discovery by LALDI-MS, in which nanomoles of the pyrene-labelled substrates and products could be detected selectively against a complex background of reagents, catalysts and additives upon evaporation of the reaction solvents. Subsequently, the group of Basak demonstrated the use of pyrene-based reagents in LALDI-MS to detect zinc ions, ${ }^{17}$ cis1,2-diols and catechols, ${ }^{18}$ and biogenic amines, ${ }^{19}$ Kita \& Kigoshi and co-workers used LALDI-MS to analyse tryptic peptides, ${ }^{20-22}$ and Guo and co-workers described pyrene-based peptide probes for the measurement of protease activity. ${ }^{23}$

Carbohydrates are the most ubiquitous and structurally diverse group of biomolecules, and their detection and analysis by mass spectrometry is notoriously difficult. ${ }^{24-27}$ Unlike proteins and nucleic acids, the biosynthesis of carbohydrates is not template-driven or under transcriptional control. Therefore, artificial amplification of a specific carbohydrate cannot be achieved and ultimately restricts the available material to the often minute heterogeneous quantities obtained from natural sources. ${ }^{26}$ Compared to peptides, carbohydrates are not easily ionised. To circumvent this issue, chemical derivatisation, such as permethylation ${ }^{28}$ or, less commonly, the addition of reducing-terminal functional groups ${ }^{29}$ is often employed to promote ionisation, enable purification and/or separation, and improve the stability and MS analysis of glycans. ${ }^{24}$ Such approaches have led MALDI-MS to be widely used for analysis and quantification of carbohydrates such as $\mathrm{N}$ - and $\mathrm{O}$-glycans. ${ }^{25,29-32}$

We hypothesised that LALDI-MS could be a suitable method for the direct detection of carbohydrates from complex biological samples. Such an approach would require the in situ derivatisation of carbohydrates with suitable LDI enhancers, which we call LALDI tags (Figure 1). Here, we report the development of water-soluble, pyrene-based LALDI tags for (in situ) labelling of reducing carbohydrates, and their subsequent detection by LALDI-MS. We have synthesised and characterised four different pyrene-based LDI enhancers, and optimised sample preparation, in order to achieve reproducible LALDI-MS measurements with good limits of detection. In addition, we have explored the use of different biorthogonal handles for the labelling of a reducing carbohydrate, lactose. Using LALDI-MS, the resulting pyrene-tagged lactose derivatives could be detected in complex aqueous samples without the need for purification, desalting or derivatisation. In addition, we have exemplified the use of LALDI tags for the direct detection (after in situ derivatisation) of lactose in cow's milk. 


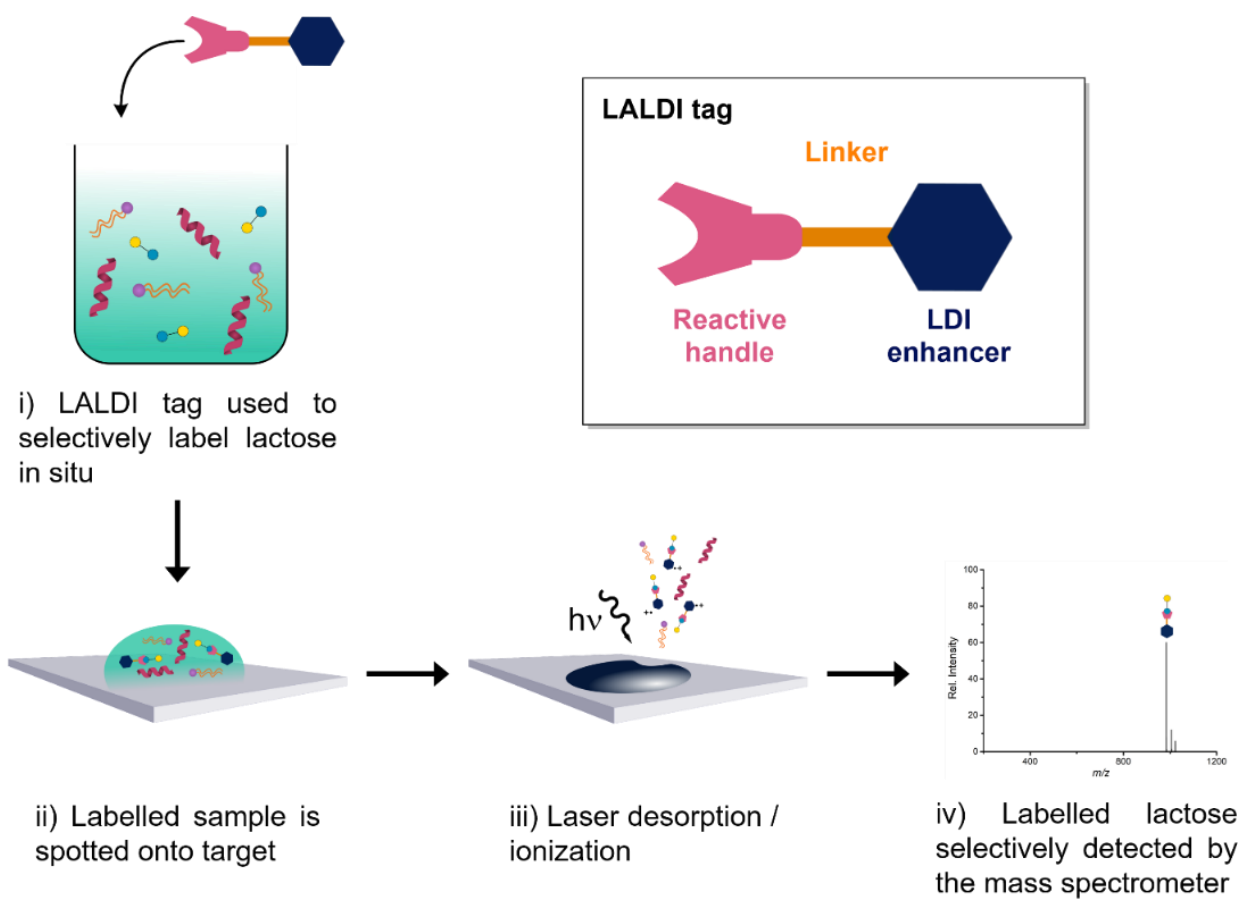

Figure 1: Concept: LALDI tags as a tool for MS-based glycomics. The figure describes the general workflow for the analysis of glycans (in this study: lactose) from a biological sample using LALDI-MS. The inset shows the general LALDI tag design, incorporating an LDI-enhancing label (dark blue) to facilitate matrix-free laser desorption/ionisation, a linker (orange) to improve aqueous solubility of the LALDI tag, and a reactive handle (pink) that provides a quick and reliable method to selectively label glycans.

\section{Results}

\section{Development of water-soluble pyrene-based ionisation enhancers}

In order to develop water-soluble LALDI tags with good sensitivity and stability in LALDI-MS, we first considered the nature of the pyrene group and its linkage to a solubilising oligoethylene glycol (OEG) linker. Most previous LALDI-MS studies used LDI enhancers based on pyrene butyramide (PyBA). ${ }^{16,18,19,23}$ In addition, Yoneda et al. reported that compounds based on 6-amidopyrene (6-APy) have increased absorption at $355 \mathrm{~nm}$ (the wavelength of lasers commonly used in MALDI instruments), and that some 6-APy compounds could be observed by LALDI-MS with limits of detection down to 10 pmol. ${ }^{20}$ However, the 6-APy tag readily fragmented to lose ketene, and the work suggested that careful design of linker chemistry is essential to avoid complex fragmentation patterns in LALDI-MS or subsequent tandem MS analysis. ${ }^{20}$ Therefore, we designed and synthesised four different pyrene-OEG conjugates for analysis in LALDI-MS. Two were based on the previously reported PyBA (1) and 6-APy (2) moieties, and two on the novel 1-amidopyrene (1-APy; 3) and pyrene urea (PyU; 4) moieties (Figure 2a). The synthesis, purification and chemical analysis of these compounds are described in the Supplementary Methods. Compounds 1 and $\mathbf{2}$ exhibited UV/vis absorption profiles identical to those reported by Yoneda et al., with 6-APy derivative $\mathbf{2}$ exhibiting a bathochromic shift of the pyrene absorption band compared to PyBA derivative $\mathbf{1}$ (Figure 2b). 1-APy derivative $\mathbf{3}$ and PyU derivative $\mathbf{4}$ exhibited similar bathochromic shifts to $\mathbf{2}$, consistent with the extended conjugation of compounds 2-4 compared to 1 . In addition, this shift was absent in an $N$-methylated variant of 3, compound S1 (see Supplementary Figure 1), suggesting that $N$-methylation results in steric hindrance preventing full conjugation between amide and pyrene. 
Compounds 1-4 were then analysed by LALDI-MS, comparing their stabilities (i.e. level of insource fragmentation), lower limits of detection and ease of spectral interpretation. A 9.4 T (Bruker solariX XR) high-performance MALDI-FT-ICR mass spectrometer was used, allowing unambiguous assignment of elemental formulae of molecular ions, cationised molecules, and decomposition products with low- or sub-ppm mass accuracy (Figure $\mathbf{2 c}, \mathbf{d}$ ).$^{33}$ Compounds were dissolved in methanol, a known amount of each sample was spotted onto a target plate, and solvent was allowed to evaporate, resulting in films containing $1 \mathrm{fmol}-100 \mathrm{pmol}$ of each compound on the target plate. LALDI-MS analysis was performed on these films without addition of any other reagents, matrices or surface materials, with three independent spots measured for each sample (see Methods for details). Measurements were initially performed using samples spotted onto standard ground steel MALDI target plates, but low shot-to-shot reproducibility was obtained (Supplementary Figure 2a). This was most likely the result of heterogeneities in the sample films. Indeed, scanning electron microscopy analysis of the sample films revealed the formation of 'coffee ring' patterns and accumulation of analytes in grooves on the target plate upon sample drying (Supplementary Figure $\mathbf{2 b}, \mathbf{c}$ ). ${ }^{34}$ Instead, use of a micro-focusing target plate designed to localise hydrophilic samples onto a smaller area ${ }^{35}$ resulted in LALDI-MS measurements with improved limits of detection of $10 \mathrm{fmol}-10 \mathrm{pmol}$, excellent shot-to-shot reproducibility, and good correlation between amount of analyte and signal intensity (Figure $\mathbf{2 c , d}$ ). Therefore, this micro-focusing target plate was used for all subsequent LALDI-MS experiments.

LALDI-mass spectra of compounds 1-4 all showed the radical cations $\left(\mathrm{M}^{\bullet+}\right)$ as the main peaks, in addition to sodiated and potassiated molecules $\left([\mathrm{M}+\mathrm{Na}]^{+},[\mathrm{M}+\mathrm{K}]^{+}\right)$(Figure $2 \mathrm{c}$ ); the latter are most likely the result of salts in the solvents and on the target plate. Because we developed LALDI tags for analyses from biological samples containing high salt concentrations, no attempts were made to suppress the detection of these cationised molecules. 6-APy 2 and 1-APy 3 achieved favourable lower limits of detection of $10 \mathrm{fmol}$ (Figure $\mathbf{2 c}$, d). However, both compounds also exhibited some in-source fragmentation. For 6-APy 2, a peak was observed at $[\mathrm{M}-42]^{*+}$ correlating with fragmentation at the terminal amide and loss of ketene, consistent with reports by Yoneda et al. ${ }^{20}$ For 1-APy 3, a peak was observed at $[\mathrm{M}-28]^{\circ+}$, which according to LC-MS analysis was not present in the synthetic product 3. Accurate mass LALDI MS (Figure 2) and product ion analysis (Supplementary Figure 3), in combination with LALDI-MS analysis of a minimal 1-APy analogue S2 (Supplementary Figures 4-6) (which also displayed $[\mathrm{M}-28]^{\circ+}$ peaks), revealed that these signals were the result of the loss of CO. Collisioninduced dissociation (CID) product ion analysis of the molecular ion of 1-APy 3 resulted in multiple expected product ions arising from fragmentations along the OEG and amide linkers (Supplementary Figure 3a). However, the $[\mathrm{M}-28]^{\circ+}$ peak observed on LALDI-MS of 1-APy 3 was absent in the CID product ion spectrum of the molecular ion of $\mathbf{3}$, suggesting that the loss of CO occurs in the MALDI source. $\mathrm{N}$-methylated 1-APy derivative $\mathbf{S 1}$ displayed reduced LDI source stability compared to 1-APy 3 , with an increased relative abundance of $[\mathrm{M}-28]^{\circ+}$ and additional fragmentation observed in the LALDI mass spectrum (Supplementary Figure 1). PyBA 1 and PyU 4 displayed little in source fragmentation and so excellent stability, and could be detected down to $100 \mathrm{fmol}$ and $1 \mathrm{pmol}$, respectively (Figure 2c,d). Qualitative comparison of compounds 1-4 in Table 1 suggests that 6-APy 2 and 1-APy $\mathbf{3}$ have the most favourable limits of detection, while PyBA 1 and PyU $\mathbf{4}$ have the highest stability. Because 1-APy derivatives are more stable (no loss of ketene) and easier to synthesise than 6-APy derivatives, and because PyBA derivatives can give complex fragmentation patterns in tandem 
MS analysis, ${ }^{20} \mathrm{LDI}$ enhancers $\mathbf{3}$ and $\mathbf{4}$ were selected for the development of LALDI tags for carbohydrate analysis.

a)
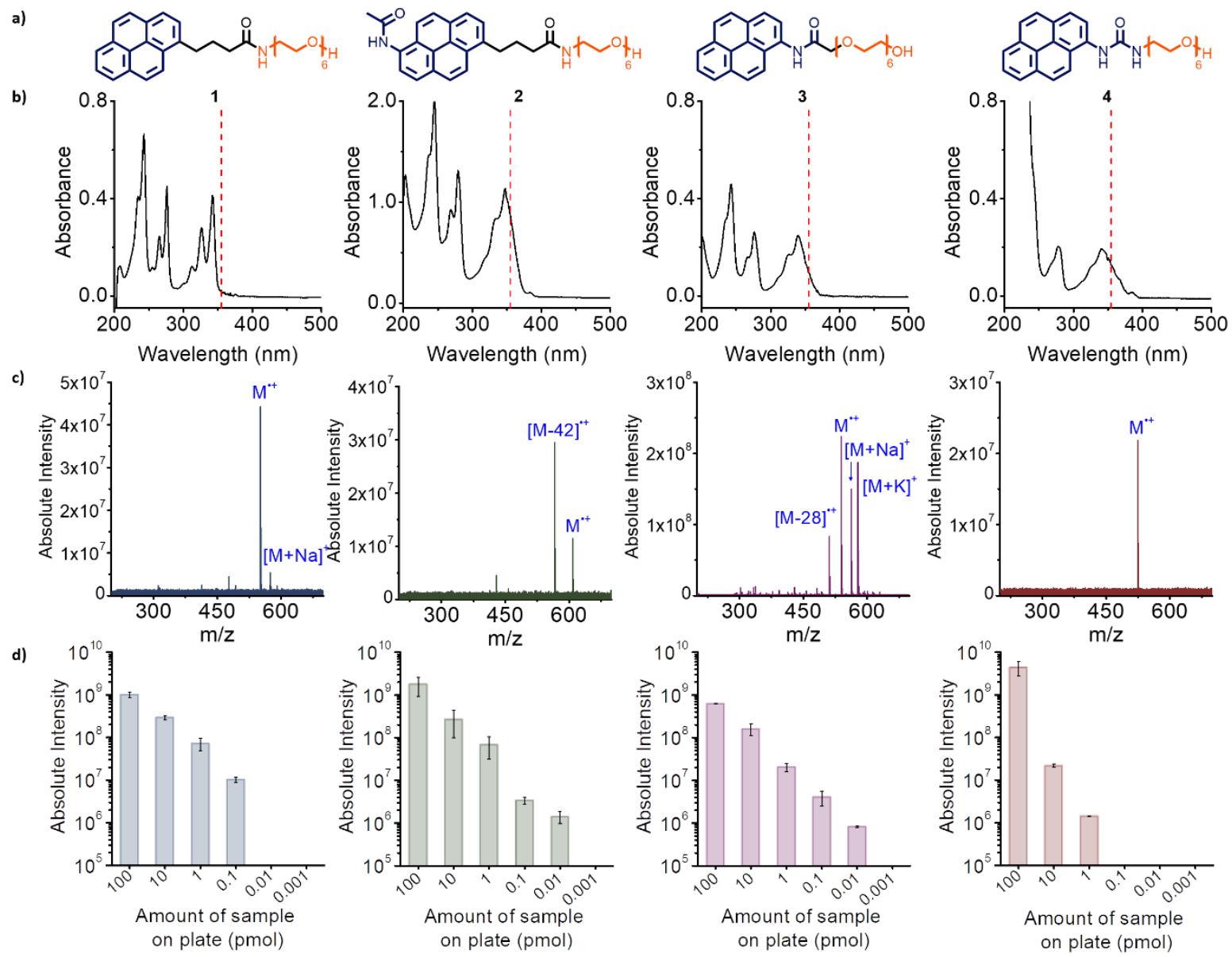

Figure 2: Performance of four different pyrene derivatives in LALDI-MS experiments. a) Structures of watersoluble pyrene-based LALDI reagents. b) UV/vis absorption spectra for compounds 1-4. The red hashed line indicates the wavelength of excitation applied in the LDI-MS instrument $(355 \mathrm{~nm})$. Note that the UV/vis spectra of pyrenes in solid state have been reported to display broad absorption bands $<391 \mathrm{~nm} .{ }^{22,36} \mathrm{c}$ ) LALDI-mass spectra of compounds 1-4 (10 pmol samples). d) Determination of limits of detection of compounds 1-4 by LALDIMS. Bars represent the mean values for the sum of all positive mode ions related to each LALDI reagent, including the molecular ions $\left(\mathrm{M}^{*+}\right)$, cationised molecules $\left([\mathrm{M}+\mathrm{Na}]^{+},[\mathrm{M}+\mathrm{K}]^{+}\right)$, and major fragments $(2 \text { : [M-ketene }]^{*+}, \mathbf{3}$ : [M$\mathrm{CO}^{\circ+}$ ) detected by LALDI-MS of decreasing sample amounts. Error bars represent the standard deviation, where $\mathrm{n}=3$ (analysis of three individual spots from the same compound solution).

Table 1: Summary of the performance of LDI enhancers 2-4 to PyBA 1 in LALDI-MS experiments. PyBA, pyrenebutyramide; 6-APy, 6-amidopyrene; 1-APy, 1-amidopyrene; PyU, pyrene urea.

\begin{tabular}{ccc}
\hline LDI enhancer & MS stability & $\begin{array}{c}\text { Limit of detection } \\
\text { (pmol) }\end{array}$ \\
\hline PyBA 1 & No major fragmentation & 0.1 \\
6-APy 2 & Additional peak at [M-42] ${ }^{++}$ & 0.01 \\
1-APy 3 & Additional peak at [M-28] & 0.01 \\
PyU 4 & No major fragmentation & 1 \\
\hline
\end{tabular}




\section{Development of LALDI tags for reducing carbohydrates}

To label reducing carbohydrates, such as $\mathrm{N}$ - or $\mathrm{O}$-glycans released through enzymatic ${ }^{37,38}$ or chemical cleavage techniques, ${ }^{39,40}$ suitable reactive handles were required that would be efficient, selective, and form a stable conjugate product in the presence of a complex biological matrix. Inspired by common glycan labelling strategies, reviewed $\mathrm{in}^{25}$, three reactive handles were chosen: hydroxylamine, ${ }^{41-44}$ hydrazide, ${ }^{45}$ and 2 -aminobenzamide (2-AB). ${ }^{46-48}$ Five unique LALDI tags were synthesised, combining the two selected LDI enhancers $\mathbf{3}$ and $\mathbf{4}$ and the three reactive handles through their solubilising linker (compounds 5-9, Table 2). The synthesis, purification and chemical analysis of these LALDI tags are described in the Supplementary Methods. Compounds 6, $\mathbf{8}$ and $\mathbf{9}$ were analysed by LALDI-MS to determine whether their detection and stability would be affected by the three different reactive handles (Supplementary Figures 7-9; Supplementary Table 1). Analysis of these unreacted LALDI tags would also provide insight relevant for the analysis of in situ labelling reactions, allowing distinctions to be made between signals relating to the products, unreacted LALDI tags, and other signals in the spectra. Each of the reagents was detected by LALDI-MS, but the compounds (especially 9) underwent more in-source fragmentation than the simple LDI enhancers 1-4. This is most likely because of the reactivity of the handles, and the instability of $\mathrm{N}-\mathrm{O}$ and $\mathrm{N}-\mathrm{N}$ bonds that we observe in our LALDI-MS experiments (see for example Supplementary Figure 10) consistent with previous reports of $\mathrm{N}-\mathrm{O}$ bond fragmentation in LALDI-MS. ${ }^{20}$

LALDI tags 5-9 were subsequently used for the labelling of lactose, which was considered an ideal model for the labelling reaction and LALDI-MS analysis because it is readily available, and because small glycans such as lactose can be difficult to detect by MALDI-MS without further chemical modifications (reviewed in ${ }^{29}$ ), use of additives, ${ }^{49,50}$ or use of specialised nanoparticle supports. ${ }^{51-53}$ Lactose was labelled with each of the five LALDI tags 5-9, with high conversion assessed by LC-MS analysis (Table 2). The pyrene-labelled lactose derivatives 10-14 were purified by preparative HPLC to enable analysis of known quantities of well-defined analytes, in order to study performance of 10-14 in LALDI-MS analysis. According to analytical HPLC, compounds 10-13 were analytically pure (Supplementary Figures 49-52), while compound 14 contained contaminants resulting from the labelling reaction that could not be removed (Supplementary Figure 53). LALDI-MS analysis was carried out on these samples (Figure 3; Table 2). 
Table 2: The use of LALDI tags 5-9 to detect lactose by LALDI-MS.

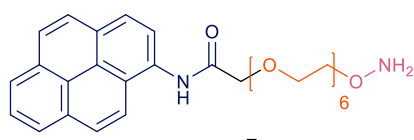

5

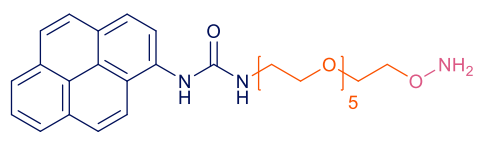

6<smiles>Nc1ccccc1C(=O)NCCOCC(=O)Nc1ccc2ccc3cccc4ccc1c2c34</smiles>

7
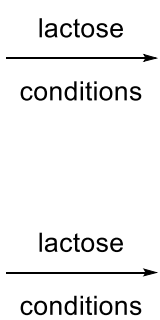

conditions

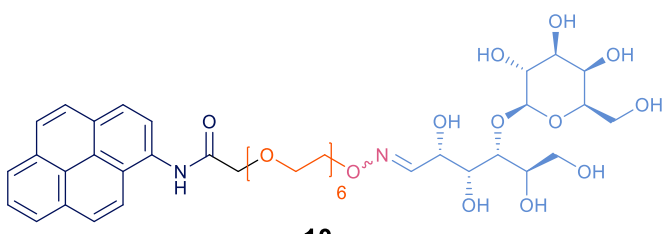

10

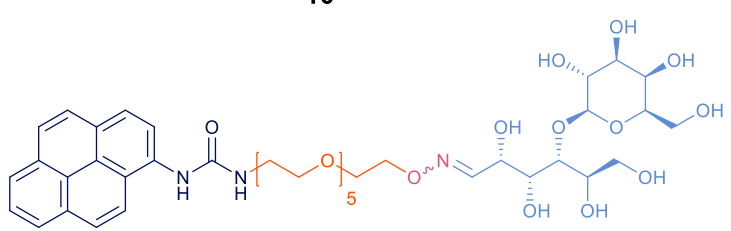

11

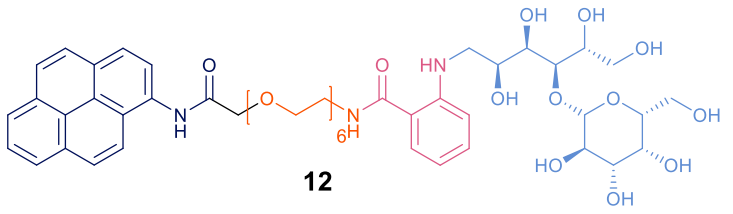

12

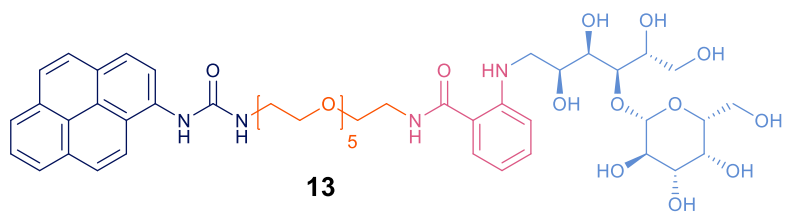

13

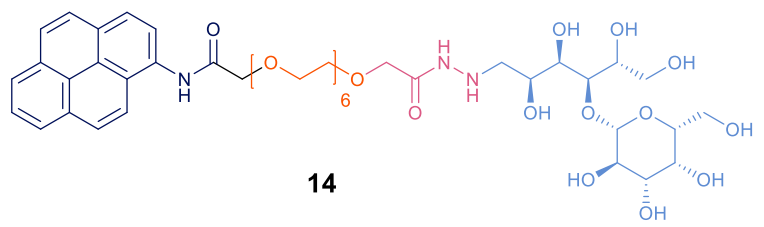

conditions

9

conditions

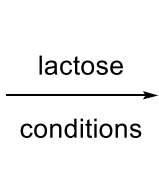

Molecular

ion MS Limit of

detected

in LALDI-

stability $¥$

detection
(pmol)

MS?

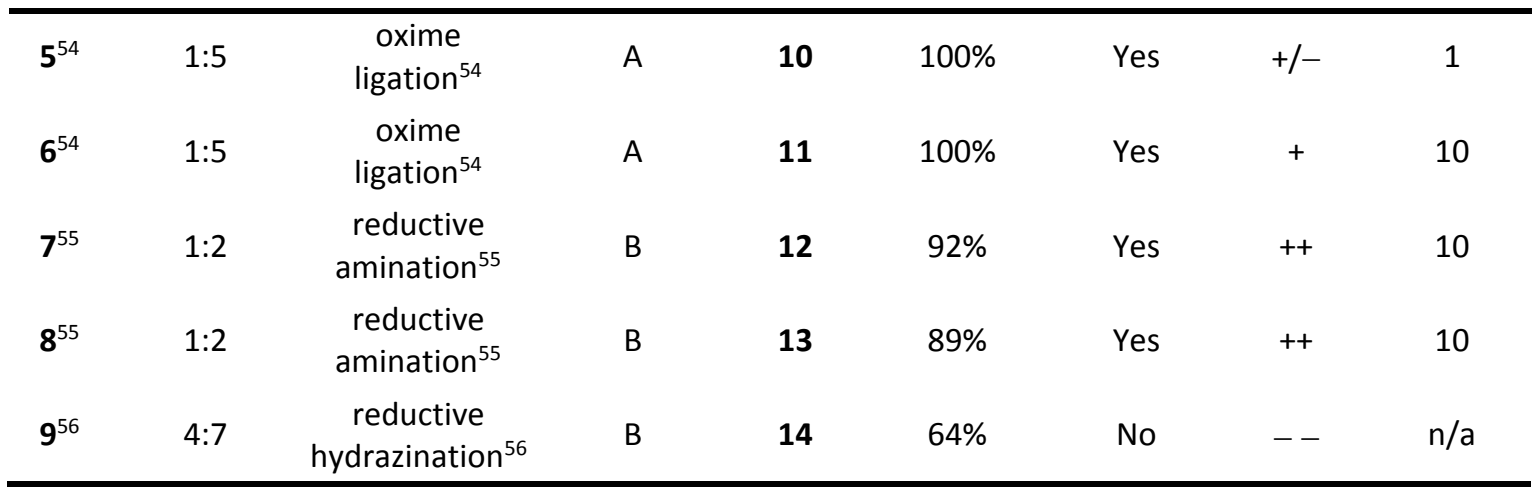

* Reaction conditions: A) 3,5-diaminobenzoic acid, MeCN : sodium citrate buffer (0.1 M, pH 3) (1:1), rt, 24 h; B) $\mathrm{NaBH}_{3} \mathrm{CN}$, DMSO:AcOH (7:3), $60^{\circ} \mathrm{C}, 2 \mathrm{~h}$. Additional details of the reaction conditions, purification and chemical analysis of the labelled lactose are described in the Supplementary Methods. + Conversion was calculated by comparing integrals of UV absorbance peaks for starting materials 5-9 and products 10-14 in the LC-MS analysis of the reaction mixture. $¥ \mathrm{MS}$ stability was assessed from i) the number of ions observed, in addition to the molecular species, on LALDI-MS analysis of each analyte, and ii) the relative abundance of the signals corresponding to the molecular ion $\left(\mathrm{M}^{*+}\right)$ and cationised molecules $\left([\mathrm{M}+\mathrm{Na}]^{+},[\mathrm{M}+\mathrm{K}]^{+}\right)$compared to the additional ions (presumably fragment ions), then scored using an arbitrary scale from ++ to -- . 


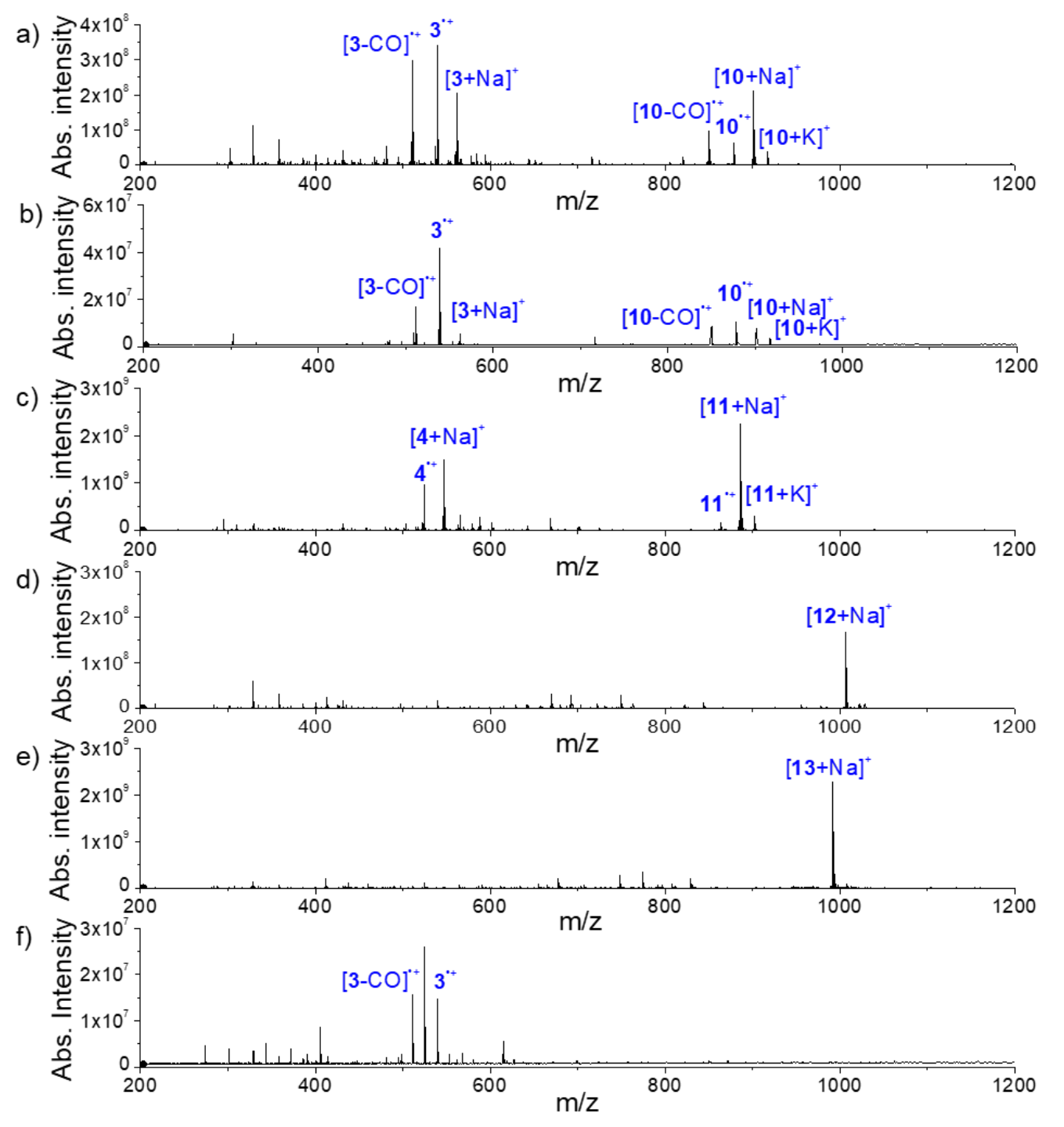

Figure 3: LALDI mass spectra for samples of LALDI-tagged lactose derivatives (100 pmol) with molecular ions, cationised molecules, and identified fragments. a) $\mathbf{1 0}$ (from MeOH). b) $\mathbf{1 0}$ (from HBSS) c) $\mathbf{1 1}$ (from MeOH). d) $\mathbf{1 2}$ (from $\mathrm{MeOH}$ ). e) $\mathbf{1 3}$ (from $\mathrm{MeOH).} \mathrm{f)} \mathbf{1 4}$ (from MeOH). HBBS = Hanks' balanced salt solution.

LALDI-MS analysis of 10-14 resulted in reproducible detection (Figure 3; Supplementary Figures 10-15). Lactose oxime derivatives 10 and 11, and the 2-AB-linked lactose derivatives 12 and 13 gave signals with good signal-to-noise ratios (Figure 3a,c-e; Supplementary Figures 10-13). However, analysis of the hydrazide-linked lactose derivative $\mathbf{1 4}$ did not yield signals for the expected products; instead the spectrum was dominated by signals for low molecular mass species, presumably produced on in-source fragmentation occurring predominantly around the hydrazide linkage (Figure 3f; Supplementary Figure 14). Consistent with analysis of simple 1-APy LDI enhancer 3 (see above), loss of $\mathrm{CO}$ was clearly detected from 1-APy-labelled lactose derivative $\mathbf{1 0}$ (Figure 3a; Supplementary Figure 10). Loss of $\mathrm{CO}$ was also observed during analysis of 1-APy-labelled lactose 12, although only low intensity signals for $[\mathbf{1 2 - C O}]^{\cdot+}$ were observed (Supplementary Figure 12). Both oxime-linked lactose derivatives $\mathbf{1 0}$ and $\mathbf{1 1}$ displayed in-source fragmentation at the oxime linkage (Figure 3a,c; Supplementary Figures 10-11). 2-AB linked lactose derivatives 12 and $\mathbf{1 3}$ were clearly detected as $[\mathrm{M}+\mathrm{Na}]^{+}$(Figure 3d,e); only low intensity fragment ions - presumably arising on fragmentation around the benzamide and secondary amine groups - were observed (Figure 3d,e; Supplementary Figures 12-13). 1-APy 10 was found to have the lowest limit of detection, observed down to a sample size of 
$1 \mathrm{pmol}$, while labelled lactose derivatives 11-13 were found to have a lower limit of detection of 10 pmol. No lower limit of detection was determined for lactose hydrazide derivative $\mathbf{1 4}$ as neither the molecular ion nor cationised molecules were detected at any sample quantity. From these results, it was concluded that pyrene-labelled lactose compounds $\mathbf{1 2}$ and $\mathbf{1 3}$ were the most stable in LALDI-MS analysis (Table 2), while compound $\mathbf{1 0}$ resulted in the lowest limit of detection (while displaying $\mathrm{N}-\mathrm{O}$ bond cleavage).

To test the detection of LALDI-tagged lactose derivatives in a complex sample matrix, LALDIMS analysis of 10-13 was repeated with the samples dissolved in the cell culture buffer Hanks' balanced salt solution (HBSS), which contains various buffer salts ([Na+ $142 \mathrm{mM},\left[\mathrm{K}^{+}\right] 5.80 \mathrm{mM},\left[\mathrm{Mg}^{2+}\right]$ $\left.0.898 \mathrm{mM},\left[\mathrm{Ca}^{2+}\right] 1.26 \mathrm{mM}\right)$, glucose $(5.55 \mathrm{mM})$, and a phenol red indicator $(26.6 \mu \mathrm{M})$. An image of the evaporated sample of LALDI-tagged lactose $\mathbf{1 0}$ in HBSS, captured by the mass spectrometer's target camera is shown in Supplementary Figure 16b. The crystalline appearance of the sample is consistent with the high abundance of buffer salts present in the samples analysed during these experiments. While the limits of detection were affected by the presence of the buffer components (Supplementary Table 2), each of the pyrene-labelled lactose derivatives 10-13 was clearly detected in the presence of HBBS, with the spectra obtained (Figure 3b; Supplementary Figure 16a) being nearly identical to those obtained from pure samples (Figure 3a,c-f). These results highlight the advantage of the LDI-enhancing label in selectively promoting ionisation and MS detection of only the labelled species, even in the presence of buffer, contaminants and high concentrations of salts.

\section{Use of a LALDI tag for selective labelling and detection of lactose in cow's milk}

To test if LALDI tags could be used to label reducing carbohydrates in situ in a complex sample matrix, LALDI tag 6 was used to label lactose directly in cow's milk by adapting the previously optimised oxime ligation conditions (Table 2). Cow's milk was chosen for this proof-of-concept because of its high lactose concentration $\left(4-5 \% \mathrm{w} / \mathrm{w}\right.$ ) and complex matrix. ${ }^{57}$ Following labelling, the sample was diluted and centrifuged to remove curd to enable the sample solution to be transferred by pipette to the MALDI plate. The supernatant was analysed directly by LALDI-MS (Figure 4), giving notably clean LALDI mass spectra displaying peaks corresponding to lactose derivative $11\left(\mathrm{~m} / \mathrm{z} 896.3591[\mathrm{M}+\mathrm{Na}]^{+}\right.$ and $\left.902.3328[\mathrm{M}+\mathrm{K}]^{+}\right)$. The most intense peak $(\mathrm{m} / \mathrm{z}$ 524.2518) was assigned as a fragment ion (11') resulting from $\mathrm{N}-\mathrm{O}$ bond cleavage. This fragment could also have been generated through fragmentation of the hydroxyl amine-functionalized LALDI tag 6 ( $N-O$ bond fragmentation was observed during LALDI-MS analysis of PyU-OEG-ONH ${ }_{2}$; see Supplementary Figure 7), but it should be noted that the amount of $\mathbf{6}$ added to the milk sample was less than the estimated amount of lactose present, and that ions corresponding to intact 6 were not detected in this LALDI-MS experiment. Although different LALDI tags and in situ labelling conditions need to be explored in future studies, these results highlight the potential of LALDI tags to be used for the labelling and analysis of reducing carbohydrates directly in complex biological samples. Furthermore, it is important to note that detection of only pyrene-containing molecules indicates that, at the concentrations tested, the LDI enhancers did not act as a matrix for the many other non-carbohydrate species present in the samples. 
a)

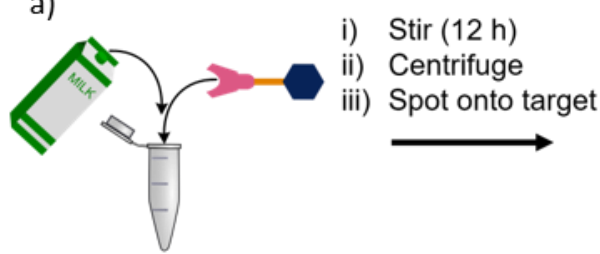

b)

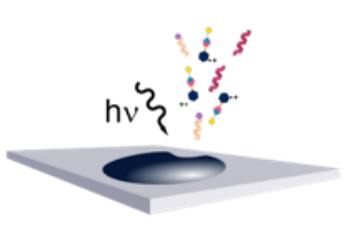

c)

)

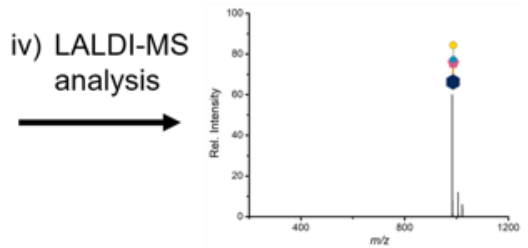

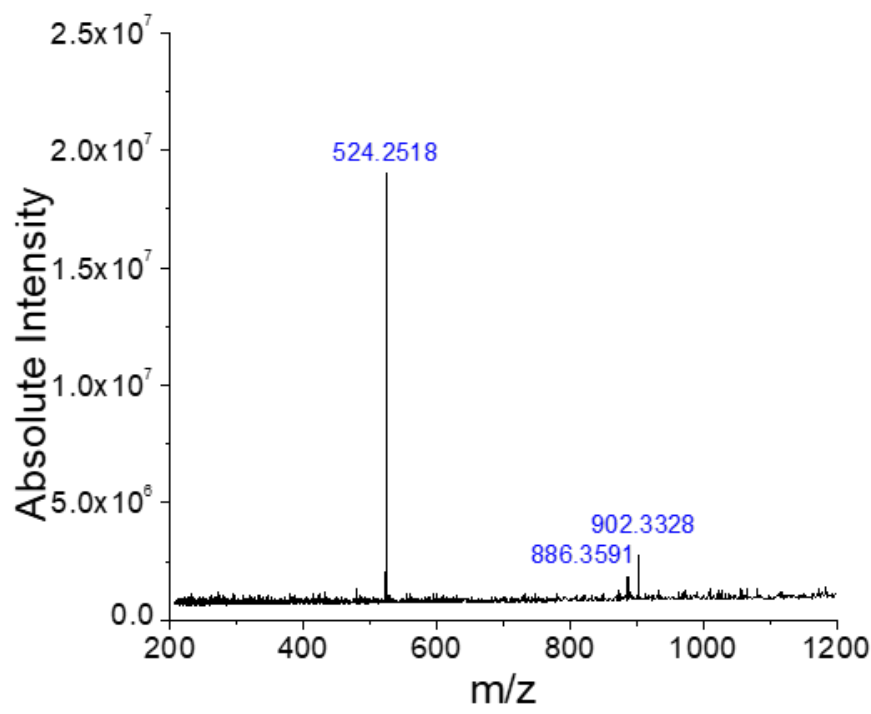

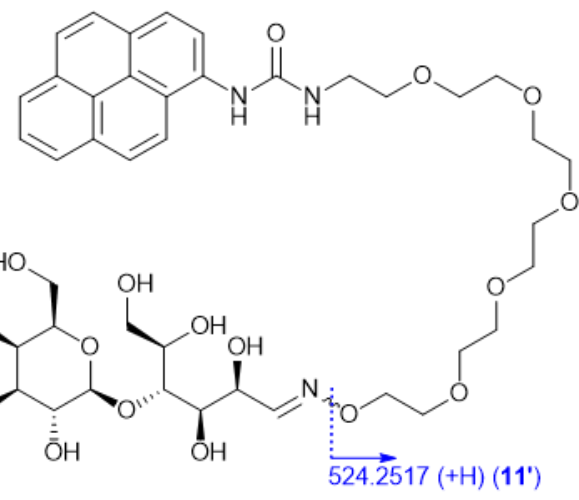

$902.3320-[\mathrm{M}+\mathrm{K}]^{+}$ $886.3580-[\mathrm{M}+\mathrm{Na}]^{+}$<smiles>O=C(NCCOCCOCCOCCOCCO)Nc1ccc2ccc3cccc4ccc1c2c34</smiles>

$11^{\prime}$

Figure 4 LALDI-MS analysis of milk following glycan labelling with LALDI tag 6. a) Schematic representation of the general workflow for in situ labelling of lactose in milk and subsequent LALDI-MS analysis. b) LALDI mass spectrum obtained following direct LALDI-MS analysis of the labelling reaction mixture; identified fragments and molecular species are annotated with their observed $\mathrm{m} / \mathrm{z}$ values. c) Chemical structures for LALDI tag-labelled lactose 11 and proposed major fragment ion 11'. Proposed assignments for fragments and molecular species observed in the LALDI mass spectrum in (b) have been annotated in the structure of 11, accompanied by their corresponding calculated monoisotopic masses.

\section{Discussion}

We have developed novel, water-soluble LDI-enhancers, exhibiting properties including low limits of detection, good stability, and predictable MS fragmentation. We introduced two new LDI enhancers, 1-APy and PyU, derivatives of which can be readily synthesised from commercially available 1-aminopyrene. By studying the behaviour of samples on different target plates, we optimised both limits of detection and reproducibility of LALDI-MS measurements. 1-APy 3 displayed lower limits of detection than PyBA derivative $\mathbf{1}$, and higher stability than 6-APy derivative $\mathbf{2}$ in LALDIMS experiments. In contrast, PyU 4 showed no in-source fragmentation but had higher limits of detection. By combining pyrene-based LDI enhancers with handles for tagging reducing carbohydrates, we were able to develop a range of water-soluble LALDI reagents capable of labelling and detecting reducing carbohydrates (in this work: lactose) by LALDI-MS. We have demonstrated that 
LALDI tagging can be performed in cow's milk, and that pyrene-tagged lactose derivatives can be detected by LALDI-MS in complex (biological) matrices without the need for further purification, chemical modification, or additional MALDI matrices.

The correlation between increased UV absorption at $355 \mathrm{~nm}$ and (slightly) improved limit of detection in LALDI-MS experiments displayed by 6-APy 2 and 1-APy 3 compared to PyBA 1 is consistent with results reported by Yoneda et al. However, the improvement in limits of detection in our experiments was modest. Moreover, PyU 4 was found to have a higher limit of detection than PyBA 1, despite displaying a similar bathochromic shift to $\mathbf{2}$ and $\mathbf{3}$. These results emphasise that factors additional to optical properties of the pyrene tag (e.g., ease of ionisation, stability of molecular ion and cationised molecules, stability to in-source fragmentation) are also likely to influence limits of detection in LALDI-MS. It should also be noted that, in LALDI-MS, samples are analysed from a dried spot, not as solutions. Sharma et al. demonstrated that, in solution, the UV absorption profile of pyrene is identical to that of PyBA derivative 1 , while solid pyrene exhibits a broader and red-shifted UV absorption profile with much higher absorbance at $355 \mathrm{~nm} .{ }^{36}$ They proposed that the observed change in absorbance was due to increased $\pi$-stacking interactions in solid pyrene. Therefore, while information gained on the solution phase UV absorbance of 1-4 was useful for comparison of our compounds to previously reported pyrene-based LDI enhancers, it may not accurately represent the absorbance of an LDI enhancer during a LALDI-MS experiment. Indeed, Kigoshi \& Kita et al. recently demonstrated that LDI enhancers based on differently substituted pyrenes (including PyBA, 6-APy and a new, highly sensitive $\mathrm{N}, \mathrm{N}$-dimethylaminopyrene) all displayed a broad absorbance band $<391 \mathrm{~nm}$ when UV/vis was performed on solid samples. ${ }^{22}$ The authors observed that the sensitivity of three pyrene derivatives in LALDI-MS was inversely correlated with their fluorescence quantum yield, suggesting that performance of LDI enhancers is related to their heat emission. This observation should be taken into account in the design of future generations of LALDI tags with increased LALDI-MS sensitivity.

LALDI-MS was preceded by the development of fluorophore-assisted laser desorption/ionisation mass spectrometry (FALDI-MS), ${ }^{58}$ in which fluorescent dyes acted as the LDI enhancers. In addition, West et al. demonstrated that 2-aminobenzamide, a common fluorescent label for reducing carbohydrates, could be used for the FALDI-MS detection of the oligosaccharide maltoheptaose. ${ }^{59}$ However, the resulting spectrum featured unidentified higher mass signals and analysis required a relatively large amount of sample ( $157 \mathrm{nmol})$. In addition, the fluorophores appeared to act as weak matrices, ionising impurities and species other than the carbohydrate analytes, thereby generating more complicated spectra than was the goal. In contrast, our results suggest that pyrene derivatives do not act as a general MALDI matrix under the conditions tested in this work, and result in ionisation of only covalently labelled species even when analysed against complex (biological) backgrounds.

Our first generation water-soluble LALDI tags for the labelling and detection of reducing carbohydrates incorporate reactive handles that are commonly used for carbohydrate derivatisation. LALDI tags with a hydroxylamine or 2-AB reactive handle were successfully used to label and detect lactose. The selection of LALDI tags for specific (biological) sample application will depend both on the compatibility of the samples with labelling conditions (e.g., solvent and $\mathrm{pH}$ ) and the required stability: the 2-AB linked products displayed higher in-source stability than oxime-linked products, with the latter undergoing $\mathrm{N}-\mathrm{O}$ fragmentation. The successful in situ labelling and detection of lactose in cow's 
milk suggests that LALDI tags can indeed be used for detection of carbohydrates in biological matrices, but it should be noted that carbohydrate ligation conditions need to be optimised further for the specific biological matrix. In addition, enrichment may be needed for the LALDI-MS analysis of less abundant carbohydrates. Interestingly, recent reports suggest that the affinity of pyrene derivatives for materials such as polystyrene ${ }^{23}$ and TSK-G3000S gel (styrene-divinylbenzene copolymer) ${ }^{21,22}$ may allow specific affinity enrichment of LALDI-tagged carbohydrates from complex mixtures before LALDIMS analysis, which could be used to significantly improve the limit of detection.

Through this investigation we have gained a better understanding of how the structure and chemical functionality of LDI enhancers, linkers, and reactive handles affect the limits of detection and in-source stability of reagents analysed by LALDI-MS. Our findings highlight LALDI tags as promising reagents for the detection/analysis of carbohydrates as well as other biomolecules from complex biological environments. Important for glycomics and activity-based profiling approaches, the reproducibility and concentration-dependence of the LALDI-MS signal intensity may additionally allow (semi-)quantitative measurements, for example through careful external calibration or internal calibration using isotopically labelled internal standards.

\section{Methods}

Synthesis and characterization. Synthetic procedures, compound characterisation and relevant spectra of final compounds are available in the Supplementary Methods.

UV/vis analysis. UV/vis absorption was measured using an Agilent Technologies Cary 100 UV-Vis Spectrophotometer. All samples were analysed as solutions in a $10 \mathrm{~mm}$ Hellma Analytics High Precision Quarts Suprasil cell. Samples for UV/vis analysis were prepared from a $1 \mathrm{mM}$ methanolic solution of the purified analytes, then adjusted to the desired concentration by serial dilution. Absorption maxima $\left(\lambda_{\max }\right)$ are given in nanometers to the nearest whole nanometer with the corresponding molar extinction coefficient $(\varepsilon)$ given in $\mathrm{M}^{-1} \mathrm{~cm}^{-1}$.

LALDI-MS analysis. LALDI-MS was carried out on a Bruker Daltonics solariX XR FTMS 9.4T mass spectrometer with an Apollo II dual ESI/MALDI ion source. All optimised analysis was performed using a Bruker Daltonics MTP AnchorChip Target 384. Ionisation/desorption of the samples was achieved using a fixed wavelength (355 $\mathrm{nm}$ ) Bruker Smartbeam II ( $\mathrm{Nd}$ :YAG) laser. $\mathrm{m} / \mathrm{z}$ values are reported to four decimal places to reflect the mass accuracies obtained using external mass calibration with phosphorus red. LALDI-MS fragmentation assignment was carried out with the assistance of a web application developed by ChemCalc that provides a list of possible molecular formulae from a given monoisotopic mass. ${ }^{60,61}$

Samples for LALDI-MS analysis were prepared from a $1 \mathrm{mM}$ solution of the purified analytes in methanol, which was then adjusted to the desired concentration with either methanol or Hanks' balanced salt solution. A known volume $(1 \mu \mathrm{L})$ of each analytical sample was spotted onto the target plate and the solvent allowed to evaporate under ambient conditions. Evaporation of aqueous solutions was assisted using reduced pressure. LALDI-MS analysis was then performed directly on each sample (without addition of further reagents such as MALDI matrix or SALDI surface material) with 1600 laser shots per acquisition (8 scans, 200 shots, $200 \mathrm{~Hz}$, medium laser focal size). Each sample was analysed in triplicate (three separate aliquots on separate targets) and the average values of the peak 
intensities are reported. Mass spectra were acquired using ftmsControl software version 2.1 (Bruker Daltonics) and processed using flexAnalysis software version 3.0 (Bruker Daltonics).

In situ labelling of lactose from milk with hydroxylamine LALDI tag 5. 3,5-diaminobenzoic acid ( $3 \mathrm{mg}$, $0.02 \mathrm{mmol}$ ) was added to a stirred solution of 3-[17-(aminooxy)-3,6,9,12,15-pentaoxaheptadecan-1yl]-1-(pyren-1-yl)urea 5 (10 mg, $0.02 \mathrm{mmol})$ in a 1:1 mixture of cow's milk: $0.1 \mathrm{M}$ citrate buffer $\mathrm{pH} 3$ $(0.5 \mathrm{~mL})$ and stirred $(12 \mathrm{~h})$. The mixture was then diluted 10 -fold with methanol, subjected to centrifugation (10000 g, $30 \mathrm{~s}$ ), and $1 \mu \mathrm{L}$ of the supernatant directly spotted onto an AnchorChip target plate, dried, and analysed by LALDI-MS.

Data availability. The authors declare that the data supporting the findings of this study are available within the article and Supplementary Information file, or from the corresponding author upon reasonable request.

\section{References}

1. Karas, M. \& Hillenkamp, F. Laser desorption ionization of proteins with molecular masses exceeding 10,000 daltons. Anal. Chem. 60, 2299-2301 (1988).

2. Tanaka, K. et al. Protein and polymer analyses up tom/z 100000 by laser ionization time-offlight mass spectrometry. Rapid Commun. Mass Spectrom. 2, 151-153 (1988).

3. El-Aneed, A., Cohen, A. \& Banoub, J. Mass spectrometry, review of the basics: electrospray, MALDI, and commonly used mass analyzers. Appl. Spectrosc. Rev. 44, 210-230 (2009).

4. Girolamo, F., Lante, I., Muraca, M. \& Putignani, L. The role of mass spectrometry in the "omics" era. Curr. Org. Chem. 17, 2891-2905 (2013).

5. Rodrigo, M. A. M. et al. MALDI-TOF MS as evolving cancer diagnostic tool: a review. J. Pharm. Biomed. Anal. 95, 245-55 (2014).

6. Fuh, M. M., Heikaus, L. \& Schlüter, H. MALDI mass spectrometry in medical research and diagnostic routine laboratories. Int. J. Mass Spectrom. 416, 96-109 (2017).

7. Duncan, M. W., Nedelkov, D., Walsh, R. \& Hattan, S. J. Applications of MALDI mass spectrometry in clinical chemistry. Clin. Chem. 62, 134-143 (2016).

8. Albrethsen, J. Reproducibility in protein profiling by MALDI-TOF mass spectrometry. Clin. Chem. 53, 852-858 (2007).

9. Duncan, M. W., Roder, H. \& Hunsucker, S. W. Quantitative matrix-assisted laser desorption/ionization mass spectrometry. Brief. Funct. Genomics Proteomics 7, 355-370 (2008).

10. Börnsen, K. O. Influence of salts, buffers, detergents, solvents, and matrices on MALDI-MS protein analysis in complex mixtures. in Mass Spectrometry of Proteins and Peptides.

Methods in Molecular Biology ${ }^{T M}$, vol 146 (ed. Chapman, J. R.) 387-404 (Humana Press, 2000).

11. Mandal, A., Singha, M., Addy, P. S. \& Basak, A. Laser desorption ionization mass spectrometry: Recent progress in matrix-free and label-assisted techniques. Mass Spectrom. Rev. 1-19 (2017).

12. Amano, J., Osanai, M., Orita, T., Sugahara, D. \& Osumi, K. Structural determination by negative-ion MALDI-QIT-TOFMSn after pyrene derivatization of variously fucosylated oligosaccharides with branched decaose cores from human milk. Glycobiology 19, 601-614 
(2009).

13. Amano, J., Sugahara, D., Osumi, K. \& Tanaka, K. K. Negative-ion MALDI-QIT-TOFMSn for structural determination of fucosylated and sialylated oligosaccharides labeled with a pyrene derivative. Glycobiology 19, 592-600 (2009).

14. Amano, J. et al. Derivatization with 1-pyrenyldiazomethane enhances ionization of glycopeptides but not peptides in matrix-assisted laser desorption/ionization mass spectrometry. Anal. Chem. 82, 8738-8743 (2010).

15. Nishikaze, T., Okumura, H., Jinmei, H. \& Amano, J. Advantages of pyrene derivatization to site-specific glycosylation analysis on MALDI mass spectrometry. Int. J. Mass Spectrom. 333, 8-14 (2013).

16. Cabrera-Pardo, J. R., Chai, D. I., Liu, S., Mrksich, M. \& Kozmin, S. A. Label-assisted mass spectrometry for the acceleration of reaction discovery and optimization. Nat. Chem. 5, 4237 (2013).

17. Addy, P. S., Basu Roy, S., Mandal, S. M. \& Basak, A. Polyaromatic label-assisted laser desorption ionization mass spectrometry (LA-LDI MS): a new analytical technique for selective detection of zinc ion. RSC Adv. 4, 23314-23318 (2014).

18. Addy, P. S., Bhattacharya, A., Mandal, S. M. \& Basak, A. Label-assisted laser desorption/ionization mass spectrometry (LA-LDI-MS): an emerging technique for rapid detection of ubiquitous cis-1,2-diol functionality. RSC Adv. 4, 46555-46560 (2014).

19. Mandal, A., Das, A. K. \& Basak, A. Label-assisted laser desorption/ionization mass spectrometry (LA-LDI-MS): use of pyrene aldehyde for detection of biogenic amines, amino acids and peptides. RSC Adv. 5, 106912-106917 (2015).

20. Yoneda, K., Hu, Y., Kita, M. \& Kigoshi, H. 6-Amidopyrene as a label-assisted laser desorption/ionization (LA-LDI) enhancing tag: development of photoaffinity pyrene derivative. Sci. Rep. 5, 17853 (2015).

21. Yoneda, K., Hu, Y., Watanabe, R., Kita, M. \& Kigoshi, H. Binding position analysis of target proteins with the use of amidopyrene probes as LA-LDI enhancing tags. Org. Biomol. Chem. 14, 8564-8569 (2016).

22. Arai, A. et al. N,N-Dimethylaminopyrene as a fluorescent affinity mass tag for ligand-binding mode analysis. Sci. Rep. 10, 1-10 (2020).

23. Ling, L., Xiao, C., Wang, S., Guo, L. \& Guo, X. A pyrene linked peptide probe for quantitative analysis of protease activity via MALDI-TOF-MS. Talanta 200, 236-241 (2019).

24. Rodrigues, J. A. et al. Mass spectrometry of carbohydrates: newer aspects. Adv. Carbohydr. Chem. Biochem. 61, 59-141 (2007).

25. Ruhaak, L. R. et al. Glycan labeling strategies and their use in identification and quantification. Anal. Bioanal. Chem. 397, 3457-3481 (2010).

26. Kailemia, M. J., Ruhaak, L. R., Lebrilla, C. B. \& Amster, I. J. Oligosaccharide analysis By mass spectrometry: a review of recent developments. Anal. Chem. 86, 196-212 (2014).

27. Ruhaak, L. R., Xu, G., Li, Q., Goonatilleke, E. \& Lebrilla, C. B. Mass spectrometry approaches to glycomic and glycoproteomic analyses. Chem. Rev. 118, 7886-7930 (2018).

28. Ciucanu, I. \& Kerek, F. A simple and rapid method for the permethylation of carbohydrates. Carbohydr. Res. 131, 209-217 (1984). 
29. Harvey, D. J. Derivatization of carbohydrates for analysis by chromatography; electrophoresis and mass spectrometry. J. Chromatogr. B Anal. Technol. Biomed. Life Sci. 879, 1196-1225 (2011).

30. Abdul Rahman, S. et al. Filter-aided N-glycan separation (FANGS): a convenient sample preparation method for mass spectrometric N-glycan profiling. J. Proteome Res. 13, 1167-76 (2014).

31. Wada, Y. et al. Comparison of the methods for profiling glycoprotein glycans-HUPO human disease glycomics/proteome initiative multi-institutional study. Glycobiology 17, 411-422 (2007).

32. Skeene, K. et al. One Filter, One Sample, and the N- and O-Glyco(proteo)me: Toward a System to Study Disorders of Protein Glycosylation. Anal. Chem. 89, 5840-5849 (2017).

33. Brown, S. C., Kruppa, G. \& Dasseux, J. L. Metabolomics applications of FT-ICR mass spectrometry. Mass Spectrom. Rev. 24, 223-231 (2005).

34. Hu, J. B., Chen, Y. C. \& Urban, P. L. Coffee-ring effects in laser desorption/ionization mass spectrometry. Anal. Chim. Acta 766, 77-82 (2013).

35. Kim, Y. Sample plate for MALDI mass spectrometry and process for manufacture of the same. WO/2006/083151 (2007).

36. Sharma, K. K., Kannikanti, G. H., Baggi, T. R. R. \& Vaidya, J. R. A pyrene formulation for fluorometric visualization of latent fingermarks. Methods Appl. Fluoresc. 6, 035004 (2018).

37. Muramatsu, T. Demonstration of an endo-glycosidase acting on a glycoprotein. J. Biol. Chem. 246, 5535-5537 (1971).

38. Plummer, T. H., Elder, J. H., Alexander, S., Phelan, A. W. \& Tarentino, A. L. Demonstration of peptide: $\mathrm{N}$-glycosidase $\mathrm{F}$ activity in endo- $\beta-\mathrm{N}$-acetylglucosaminidase $\mathrm{F}$ preparations. J. Biol. Chem. 259, 10700-10704 (1984).

39. Merry, A. H. et al. Recovery of intact 2-aminobenzamide-labeled O-glycans released from glycoproteins by hydrazinolysis. Anal. Biochem. 304, 91-99 (2002).

40. Rademaker, G. J. et al. Mass spectrometric determination of the sites of O-glycan attachment with low picomolar sensitivity. Anal. Biochem. 257, 149-160 (1998).

41. Ulrich, S., Boturyn, D., Marra, A., Renaudet, O. \& Dumy, P. Oxime ligation: A chemoselective click-type reaction for accessing multifunctional biomolecular constructs. Chem. - A Eur. J. 20, 34-41 (2014).

42. Ewan, H. S. et al. Synthesis of sugar oxime ether surfactants. Tetrahedron Lett. 55, 4962-4965 (2014).

43. McReynolds, K. D., Dimas, D. \& Le, H. Synthesis of hydrophilic aminooxy linkers and multivalent cores for chemoselective aldehyde/ketone conjugation. Tetrahedron Lett. 55, 2270-2273 (2014).

44. Dell, A., Oates, J. E., Morris, H. R. \& Egge, H. Structure determination of carbohydrates and glycosphingolipids by fast atom bombardment mass spectrometry. Int. J. Mass Spectrom. Ion Phys. 46, 415-418 (1983).

45. Avigad, G. Dansyl hydrazine as a fluorimetric reagent for thin-layer chromatographic analysis of reducing sugars. J. Chromatogr. A 139, 343-347 (1977). 
46. Zhou, S., Veillon, L., Dong, X., Huang, Y. \& Mechref, Y. Direct comparison of derivatization strategies for LC-MS/MS analysis of N-glycans. Analyst 142, 4446-4455 (2017).

47. Locke, D. et al. Neutral, acidic, and basic derivatives of anthranilamide that confer different formal charge to reducing oligosaccharides. Carbohydr. Res. 339, 221-231 (2004).

48. Keser, T., Pavić, T., Lauc, G. \& Gornik, O. Comparison of 2-aminobenzamide, procainamide and RapiFluor-MS as derivatizing agents for high-throughput HILIC-UPLC-FLR-MS N-glycan analysis. Front. Chem. 6, 1-12 (2018).

49. Laremore, T. N. \& Linhardt, R. J. Improved matrix-assisted laser desorption/ionization mass spectrometric detection of glycosaminoglycan disaccharides as cesium salts. Rapid Commun. Mass Spectrom. 21, 1315-1320 (2007).

50. Yamagaki, T., Suzuki, H. \& Tachibana, K. Solid-phase fluorescence and ionization efficiency in negative-ion matrix-assisted laser desorption/ionization of neutral oligosaccharides: interaction between $\beta$-carboline matrix and ammonium salt. J. Am. Soc. Mass Spectrom. 18, 714-723 (2007).

51. Su, C. L. \& Tseng, W. L. Gold nanoparticles as assisted matrix for determining neutral small carbohydrates through laser desorption/ionization time-of-flight mass spectrometry. Anal. Chem. 79, 1626-1633 (2007).

52. Watanabe, T., Kawasaki, H., Yonezawa, T. \& Arakawa, R. Surface-assisted laser desorption/ionization mass spectrometry (SALDI-MS) of low molecular weight organic compounds and synthetic polymers using zinc oxide $(\mathrm{ZnO})$ nanoparticles. J. Mass Spectrom. 43, 1063-1071 (2008).

53. Wu, C. L. et al. Selective enhancement of carbohydrate ion abundances by diamond nanoparticles for mass spectrometric analysis. Anal. Chem. 85, 3836-3841 (2013).

54. Crisalli, P. \& Kool, E. T. Water-soluble organocatalysts for hydrazone and oxime formation. J. Org. Chem. 78, 1184-1189 (2013).

55. Bigge, J. C. et al. Nonselective and efficient fluorescent labeling of glycans using 2-amino benzamide and anthranilic acid. Anal. Biochem. 230, 229-238 (1995).

56. Grün, C. H. et al. One-step biotinylation procedure for carbohydrates to study carbohydrateprotein interactions. Anal. Biochem. 354, 54-63 (2006).

57. Scrimshaw, N. S. \& Murray, E. B. The acceptability of milk and milk products in populations with a high prevalence of lactose intolerance: Chapter 3 Lactose content of milk and milk product. Am. J. Clin. Nutr. 48, 1099-1104 (1988).

58. West, R. E., Findsen, E. W. \& Isailovic, D. Fluorophore-assisted laser desorption/ionizationmass spectrometry (FALDI-MS). Int. J. Mass Spectrom. 353, 54-59 (2013).

59. West, R. E., Jacobs, J. B. \& Isailovic, D. The analyses of fluorescently labeled biomolecules using ultraviolet-wavelength fluorophore-assisted laser desorption/ionization-mass spectrometry (UV FALDI-MS). Int. J. Mass Spectrom. 389, 39-46 (2015).

60. Patiny, L. ChemCalc: Molecular formula from monisotopic mass.

61. Patiny, L. \& Borel, A. ChemCalc: A building block for tomorrow's chemical infrastructure. J. Chem. Inf. Model. 53, 1223-1228 (2013). 


\section{Acknowledgements}

This work was supported by a University of Leeds PhD studentship to J.R.H, and an EPSRC Core Capability Grant (EP/K039202/1). The FTMS analyses were carried out at the University of York Centre of Excellence in Mass Spectrometry, which was created thanks to a major capital investment through Science City York, supported by Yorkshire Forward with funds from the Northern Way Initiative, and subsequent support from EPSRC (EP/K039660/1; EP/M028127/1). We thank Bruker Daltonics for the donation of the ground-steel MALDI target plate used for scanning electron microscopy analysis, and Matthew Broadbent for cutting the plate to size.

\section{Author contributions}

J.R.H. carried out synthesis and characterisation of reagents, and UV/vis and LALDI mass spectrometry experiments. E.T.B. contributed to LALDI mass spectrometry experiments. A.N.K. performed electron microscopy experiments. J.R.H, S.L.W., J.T.-O. and R.S.B. designed experiments. J.R.H. and A.N.K. analysed data. J.R.H. made figures. S.L.W., J.T.-O. and R.S.B. provided project supervision. R.S.B. conceived the project. J.R.H., J.T-O. and R.S.B. wrote the manuscript. All authors commented on the manuscript.

\section{Conflict of interest}

The authors declare no conflict of interest.

\section{Additional Information}

Supplementary Information (Supplementary Figures, Methods and References, including details of the synthesis and characterisation of compounds) 